\title{
How solid surface free energy determines coalescence-induced nanodroplet jumping: a molecular dynamics investigation
}

\author{
Qiang Sheng ${ }^{\mathrm{a}}$, Jie Sun $^{\mathrm{b} *}$, Wen Wang ${ }^{\mathrm{a}}$, Hua Sheng Wang ${ }^{\mathrm{a} *}$ and Colin G. Bailey ${ }^{\mathrm{a}}$ \\ ${ }^{a}$ School of Engineering and Materials Science, Queen Mary University of London, London E1 4NS, UK \\ ${ }^{\mathrm{b}}$ School of Chemical Engineering and Technology, Xi'an Jiaotong University, Xi'an, Shaanxi 710049, China
}

\begin{abstract}
Molecular dynamics simulation is performed to investigate how solid surface free energy determines the coalescence-induced jumping of nanodroplet on superhydrophobic surfaces. The nanodroplet-jumping is found highly sensitive to the solid surface free energy represented by the fluid-solid bonding strength parameter $\beta$. The coalesced-nanodroplet fails to jump off the surface when $\beta$ is 0.15 (contact angle being $145^{\circ}$ ) while succeeds to jump off the surface when $\beta$ is 0.05 (contact angle being $175^{\circ}$ ). We find that a small proportion (ca. 2 4\%) of the surface free energy released in both cases is eventually converted to kinetic energy in the jumping direction, which is in the same order as the conversion efficiency previously predicted for microdroplets. A lower solid surface free energy decreases viscous and interfacial dissipation and hence increases the kinetic energy converted and eventually leads to sufficient kinetic energy in the jumping direction for nanodroplet to jump up. Our results also address the importance of the liquid-solid surface interaction in the coalescence-induced jumping of nanodroplets and the determination of the minimum size of jumping nanodroplets.
\end{abstract}

KEYWORDS: Molecular dynamics simulation; Nanodroplet jumping; Superhydrophobic surface; Surface free energy

\section{INTRODUCTION}

The coalescence of two or more droplets drives the coalesced droplet to jump off customized superhydrophobic surfaces. This is termed as the coalescence-induced droplet jumping. ${ }^{1,2}$ The excess surface

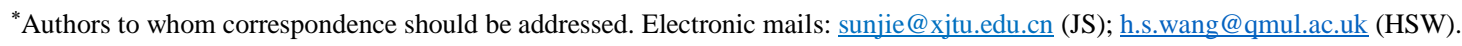


free energy of droplets, released by the reduction of surface area throughout coalescence, is converted to kinetic energy of the droplet normal to the substrate surface. ${ }^{3-5}$ This phenomenon has been widely investigated for complex interfacial physics and potential applications in self-cleaning, ${ }^{6-7}$ anti-icing ${ }^{8-9}$ and heat transfer performance enhancement ${ }^{10-11}$ experimentally, ${ }^{4,12-23}$ theoretically ${ }^{13,24-25}$ and numerically..$^{3-5,26-34}$

The conversion efficiency of the surface free energy released to kinetic energy is affected by many factors, including droplet size, solid surface adhesion, contact angle hysteresis and wetting status. It has been demonstrated that the droplet size has an important effect on the energy conversion because both the surface free energy released and the viscous dissipation depend on the droplet size. The relative importance between viscous dissipation and released surface free energy can be described by Ohnesorge number ( $O h)$, as $O h=\mu / \sqrt{\rho \gamma_{\mathrm{lv}} r}$, where $\mu, \rho, \gamma_{\mathrm{lv}}$ and $r$ being the dynamic viscosity, density, surface tension and radius of droplets, respectively. A theoretical analysis for the droplet coalescence on a rough superhydrophobic surface established an analytical correlation among the coalescence-induced velocity, solid surface free energy, viscous dissipation and droplet size. ${ }^{24}$ They showed that the self-propelled behavior occurs only for droplets of the radius within a certain range. In addition, as the droplet size decreases, the jumping velocity increases, reaches the maximum value at the diameter of ca. $50 \mu \mathrm{m}$, and then decreases. ${ }^{24}$ The decrease in jumping velocity of small size droplets is due to the increasing viscous effect and hence the decreasing conversion efficiency of released surface free energy. Earlier numerical simulations ${ }^{25}$ and theoretical modelling ${ }^{12,24,26}$ predicted that nanoscale coalesced-droplets are unable to jump up because the released surface free energy is largely consumed by considerable viscous dissipation. This is also predicted by a theoretical analysis based on the conservation of droplet interfacial free energy and viscous dissipation before and after two condensed droplets merge. ${ }^{25}$ They found that a merged droplet of too small or too large size is unable to jump up because of the domination of viscous dissipation or gravity, respectively.

However, some other investigations $s^{4,30,33,35}$ have demonstrated that the size of self-jumping droplets can be downsized to nanoscale. Recently a molecular dynamics simulation ${ }^{30}$ demonstrated that despite the large internal viscous dissipation, the coalesced nanodroplet on a super-hydrophobic surface can jump up, with a contact angle of approximately 180 degree. ${ }^{30}$ The coalescence-induced jumping of water nanodroplet (radius $R \approx 500 \mathrm{~nm}$ ) was experimentally investigated on superhydrophobic surfaces of carbon nanotubes by varying the thickness of the conformal hydrophobic coating. ${ }^{35}$ They showed that the minimum radius of 
jumping nanodroplets increases with increasing solid fraction and decreasing apparent advancing contact angle.

The coalescence-induced jumping of the droplets has been explained by the bouncing force of the solid surface. However, at nanoscale, the liquid-solid interaction has considerable effect on the movement of the contact line during the dewetting process..$^{31,36-38}$ In the condensation on lotus leaves, for example, the interfacial dissipation could be an important factor to control the jumping of nanodroplet. ${ }^{12}$ The solid surface free energy (i.e. fluid-solid interfacial free energy) also influences the conversion of the released surface free energy (vapor-liquid interfacial free energy) to the kinetic energy of droplets. Moreover, the modelling of the moving contact line encounters a problem of singularity behavior and thus the Navier-slip model, geometric formulation method or even zero-adhesion assumption are employed to avoid this problem. ${ }^{33} \mathrm{~A}$ theoretical analysis has shown that the key factors, resulting in condensed droplets jumping, include the resistance to the contact line moving on solid surfaces in addition to the released surface free energy. ${ }^{25}$ The experimental investigation explored the limitation to nanodroplet jumping due to surface adhesion in addition to the hydrodynamic limitation due to viscous dissipation. ${ }^{35}$ They investigated condensation on carbon nanotube surfaces with an effective solid fraction of about 0.10 and found that surface adhesion plays a vital role in determining the minimum size of jumping nanodroplets. ${ }^{35}$ It can be seen that the mechanism of nanodroplet jumping, particularly the adhesion effect at the liquid-solid interface at nanoscale, is still worthy of further investigation.

In the present work, molecular dynamics simulation is performed to investigate the coalescence and jumping of nanodroplet on superhydrophobic surfaces with different surface free energies. The moving contact line is directly monitored by detecting the molecules at the liquid-solid and liquid-vapor interfaces. The variations of potential energy of molecules at interfaces are analyzed. Particularly, we investigate the mechanism of solid surface free energy during the coalescence and jumping processes by comparing the jumping and non-jumping cases.

\section{SIMULATION DETAILS}

\section{A. Simulation System}


In the molecular dynamics simulation, the fluid-fluid molecular interaction is described by the Lennard-Jones (L-J) potential function $\varphi(r)=4 \varepsilon\left[(\sigma / r)^{12}-(\sigma / r)^{6}\right]$, where $r$ is the intermolecular separation, $\sigma$ is the length scale and $\varepsilon$ is the energy scale, for argon, $\sigma=0.314 \mathrm{~nm}$ and $\varepsilon=1.656 \times 10^{-21} \mathrm{~J}$. The function is truncated at a cut-off radius $r_{\mathrm{c}}=4.0 \sigma$, beyond which molecular interactions are neglected. The simulation system, as shown in Fig. 1, consists of two nanodroplets on a solid surface in a simulation box with the size of $l_{x} \times l_{y} \times l_{z}=105 \sigma \times 74 \sigma \times 155 \sigma$. Each nanodroplet has a radius of $r=25 \sigma$ (a diameter of $17 \mathrm{~nm}$ ), containing about 47500 molecules. The semi-infinite solid surface at the bottom is represented by three layers of atoms forming a (111) plane of a face-centered cubic lattice with the lattice constant $\sigma_{\mathrm{s}}=0.814 \sigma$. Neighboring solid atoms are connected by Hookean springs with the constant $k=3249.1 \varepsilon \sigma^{-2} \cdot{ }^{39}$ For temperature control, two extra layers of solid atoms are set below the three layers. The lower layer is stationary as a frame while the upper is governed by the Langevin thermostat $\frac{\mathrm{d} \mathbf{p}_{\mathrm{i}}}{\mathrm{d} t}=-\alpha \mathbf{p}_{\mathrm{i}}+\mathbf{f}_{\mathrm{i}}+\mathbf{F}_{\mathrm{i}}$, where $\alpha=168.3 \tau^{-1}$ is the damping constant, ${ }^{40} \mathbf{p}_{\mathrm{i}}$ is the momentum of the $i$ th solid molecule; $\mathbf{f}_{\mathrm{i}}$ is the sum of the forces acting on the $i$ th solid molecule, $\mathbf{F}_{\mathrm{i}}$ is a random force, of which each component is sampled to form the Gaussian distribution with zero mean value and variance $2 \alpha k_{\mathrm{B}} T_{s} / \delta t$ ( $k_{\mathrm{B}}$ is the Boltzmann constant and $\delta t=0.01 \tau$ is the time step, where $\tau=\sqrt{m \sigma^{2} / \varepsilon}$ is the characteristic time, $m$ being the mass of a fluid molecule). This technique of constant temperature control is feasible for both fluid ${ }^{41,42}$ and solid. ${ }^{39,40}$ The fluid-solid interaction is also described by the L-J potential function but with a different length scale $\sigma_{\mathrm{fs}}=0.91 \sigma$ and a different energy scale $\varepsilon_{\mathrm{fs}}=\beta \varepsilon$, where the fluid-solid bonding strength parameter $\beta$ measures the wettability: the values set here are $\beta=0.15$ and $\beta=0.05$, corresponding to contact angles of $145^{\circ}$ and $175^{\circ}$, respectively, according to our earlier investigations..$^{43,44}$ The description of wettability based on fluid-solid interaction strength has been widely employed to simulate the dependence of surface wettability on solid surface free energy ${ }^{40,42-44}$. In our previous work ${ }^{43}$, when $\beta$ increases from 0.10 to 0.70 , the contact angle decreases from $175^{\circ}$ to $20^{\circ}$, at temperature of $T=0.90 \varepsilon k_{B}^{-1}$. The surface wettability exhibits transition from superhydrophoblic, hydrophobic, hydrophilic to superhydrophilic. 
In the initialization of the simulation, liquid molecules are arranged in two nanodroplets, according to the saturation liquid density $\left(\rho_{\mathrm{s}}\right)$ at $T_{\mathrm{v}}=0.75 \varepsilon k_{\mathrm{B}}^{-1}$. Vapor molecules are arranged in the rest of the simulation box according to the vapor density $\rho_{\mathrm{v}}$. Periodic boundary conditions are employed at the sides and a diffuse reflection boundary condition is employed at the top end. Prior to the onset of coalescence, a period of $100 \tau$ is allowed for the simulation system to reach a thermal equilibrium state. To prevent the two nanodroplets from coalescing, an artificial reflective boundary condition, parallel to the $y-z$ plane, is set at $x$ $=0$ between the two nanodroplets before the coalescence commences. The liquid molecules colliding with the artificial surface are reflected by reversing the $x$-velocity. When the system reaches an equilibrium state, the artificial boundary condition is removed at $t=0 \tau$. The nanodroplets start coalescing and a period of $400 \tau$ is allowed for the evolution. The temperature control of the fluid is turned off but the temperature of the solid surface is still maintained at $T_{\mathrm{v}}=0.75 \varepsilon k_{\mathrm{B}}^{-1}$.

\section{B. Interface detection method}

The densities of liquid and vapor are defined macroscopically. To analyze the interfacial phenomenon, it is necessary to identify liquid and vapor molecules as well as the molecules at the liquidvapor interface and the liquid-solid interface. The number of interactions of a fluid molecule with other molecules determines the level of molecular potential energy. Thus, for a fluid molecule, the number of molecules within a sphere of a reference radius $R$ is calculated as coordination number, $N_{\text {co }}$. Wolde and Frenkel $^{45}$ used a reference radius $R=1.5 \sigma$ and found that the molecules with $N_{\text {co }}>4$ can be detected as liquid molecules.

The detection method is illustrated in a liquid-vapor coexistence system as shown in Fig. 2(a). A liquid film attached onto the solid surface is in a thermal equilibrium state with the saturated vapor at temperature of $T_{\mathrm{v}}=0.75 \varepsilon k_{\mathrm{B}}^{-1}$. The size of the system is $l_{x} \times l_{y} \times l_{z}=40 \sigma \times 40 \sigma \times 100 \sigma$. The initial thickness of the liquid film is taken to be $28 \sigma$. The liquid molecules interacting with the solid surface are essentially a layer of liquid molecules attached to the solid surface. Its thickness is the cutoff radius of liquidsolid interaction i.e. $4.0 \sigma_{\mathrm{fs}}$. The liquid molecules confined in this layer are detected as the molecules at the liquid-solid interface (ls molecules). To detect the molecules at the liquid-vapor interface (lv molecules), we calculate $N_{\text {co }}$ of all the fluid molecules using a sphere of a reference radius $R=3.75 \sigma$. The distributions of 
density $\rho$ and $N_{\text {co }}$ (in a certain range across the liquid-vapor interface), perpendicular to the liquid-vapor interface, are shown in Fig. 2(b). The coordination number of bulk liquid molecules is high while that of the bulk vapor molecules is low. It is assumed that the lv molecules have a coordination number between two critical values i.e. $N_{\mathrm{co}, 1}<N_{\mathrm{co}}<N_{\mathrm{co}, 2}$. The two critical values $N_{\mathrm{co}, 1}$ and $N_{\mathrm{co}, 2}$ are determined below. In Fig. 2(b), the density at $z / z_{0}=0.028$ is equal to the average density of bulk liquid and bulk vapor. The surface at $z / z_{0}=0.028$ is regarded as the boundary between the liquid and vapor. The corresponding $N_{\text {co }}\left(N_{\text {co }}=100\right)$ is determined as the critical coordination number $N_{\text {co, } 1}$. The critical coordination number $N_{\mathrm{co}, 2}$ is determined by trial and error method. The number of the detected lv molecules is calculated as $N_{\mathrm{lv}}$ and the number density per unit area $\left(n_{\mathrm{lv}}\right)$ is calculated as $n_{\mathrm{lv}}=N_{\mathrm{lv}} / A$, where $A$ is the area of the liquidvapor interface. The dependence of $n_{\mathrm{lv}}$ on value of $N_{\mathrm{co}, 2}$ is shown in Fig. 2(c). As $N_{\mathrm{co}, 2}$ increases, $n_{\mathrm{lv}}$ firstly increases gradually and then increases rapidly after $N_{\mathrm{co}, 2}=158$. When $N_{\mathrm{co}, 2}$ is less than $158, n_{\mathrm{lv}}$ is underestimated because the lv molecules near the bulk liquid molecules are neglected. However, when $N_{\mathrm{co}, 2}$ is slightly larger than 158 , an overestimated $n_{\mathrm{lv}}$ is seen because some molecules in the bulk liquid are detected as lv molecules (see in Fig. 2(d)). Therefore, the critical coordination number $N_{\mathrm{co}, 2}$ is determined to be 158. In Fig. 2(e), the liquid molecules with $100<N_{\text {co }}<158$ are detected as the lv molecules while the rest of the molecules are detected as the molecules in bulk liquid (ll molecules), as shown in yellow and green respectively. This detection method based on coordination number can also be employed to detect the liquid molecules at curved liquid-vapor interface.

\section{RESULTS AND DISSCUSSION}

As shown in Fig. 3, the processes in both cases appear three successive stages, namely the coalescence $\left(t<t_{\mathrm{a}}\right)$, restoration $\left(t_{\mathrm{a}}<t<t_{\mathrm{b}}\right)$ and oscillation or jumping $\left(t>t_{\mathrm{b}}\right)$ stages. Once the coalescence commences, a liquid bridge forms immediately and expands quickly. The wetting leads to a circular liquidsolid contact area at $t_{\mathrm{a}}$. The contact line recedes in both $x$-direction and $y$-direction until $t_{\mathrm{b}}$. After $t_{\mathrm{b}}$, different evolutions are observed in the two cases. On the solid surface with $\beta=0.15$, the coalesced- 
nanodroplet does not jump up but oscillates instead. On the contrary, on the solid surface with $\beta=0.05$, the nanodroplet jumps off the solid surface.

The differences between the two cases are quantitatively analyzed in detail. The time-evolutions of the position of the mass center of the nanodroplet in the $z$-direction $\left(z_{\mathrm{c}}\right)$ and the velocity of its mass center $\left(v_{\mathrm{c}}\right)$ are plotted in Figs. 4(a) and (b), respectively. Figures 4(c) and (d) illustrate the momentum of the coalescing nanodroplet and its components in the $z$-direction and -z-direction, respectively. Furthermore, the liquid-solid contact area shown in Fig. 5 is quantified. The size scales of the liquid-solid contact area are monitored in the $x$ - and $y$-directions $\left(B_{x}\right.$ and $\left.B_{y}\right)$ and the moving velocities of the contact line $\left(v_{\mathrm{B}, x}\right.$ and $\left.v_{\mathrm{B}, y}\right)$ are also calculated, as shown in Fig. 6.

\section{A. Coalescence stage $\left(t<t_{\mathrm{a}}\right)$}

\section{On surface with $\beta=0.15$}

Initially, a clearly circular liquid-solid contact area is observed for both nanodroplets. With the onset of coalescence, as shown in Fig. 3, a liquid bridge forms, connecting the two nanodroplets, and expands quickly in the $z$-direction. The mass center descends and its downwards velocity increases in the -z-direction until $35 \tau$. Then, the liquid bridge touches the solid surface, resulting in a peanut-like shape of the liquidsolid contact area. The changes of the numbers of the lv molecules $\left(\Delta N_{\mathrm{lv}}\right)$, ls molecules $\left(\Delta N_{\mathrm{ls}}\right)$ and 11 molecules $\left(\Delta N_{11}\right)$ are calculated in comparison to their values at $t=0$. Their evolutions with time for both cases are shown in Figs. 7(a) and (b), respectively.

Actually, $\Delta N_{\mathrm{lv}}$ and $\Delta N_{\mathrm{ls}}$ can represent the area changes of the liquid-vapor and liquid-solid interfaces. The touch to the solid surface of the liquid bridge flow leads to an increase in liquid-solid contact area in the initial period of coalescence stage. This was also observed in the earlier numerical simulation of microdroplets. ${ }^{31}$ As shown in Fig. 6, the contact line does not appear noticeable moving prior to $35 \tau$. After $35 \tau$, the solid surface counteracts the impingement of the liquid bridge flow, forcing a fraction of the moving mass towards the $y$-direction and hence leading to a decrease in $B_{x}$ and an increase in $B_{y}$.

Prior to $67 \tau, z_{\mathrm{c}}$ keeps lowering but the downwards $v_{\mathrm{c}}$ starts to decrease, as shown in Fig. 4 . The liquid-solid contact area reaches an elliptical shape at $67 \tau$, when $v_{\mathrm{c}}$ decreases to zero, as shown in Fig. 5(a). 
After $67 \tau$, the velocity of the mass center $v_{\mathrm{c}}$ becomes upwards. During the acceleration, the magnitude of the bouncing force acting on the nanodroplet is indicated by the slope of momentum variation as marked in Fig. 4 (c). As shown in Fig. 6, the contact line keeps receding in the $x$-direction and advancing in the $y$ direction. At $t_{\mathrm{a}}=108 \tau$, when $B_{x}$ is equal to $B_{y}$, the liquid-solid contact area reaches a circular shape (see Fig. 5(a)), illustrating that the coalesced-nanodroplet has transformed from a peanut-like shape to a spherical crown shape. This marks the end of the coalescence stage.

\section{On surface with $\beta=0.05$}

Compared with the case of $\beta=0.15$, the initial values of $B_{x}$ and $B_{y}$ are noticeably lower, indicating that the nanodroplet on the super-hydrophobic surface has a smaller liquid-solid contact area, as shown in Figs. 5 and 6. When the coalescence commences, as shown in Fig. $4, z_{\mathrm{c}}$ keeps almost unchanged at the beginning and no appreciable movement of the mass center is observed. $v_{\mathrm{c}}$ starts increasing apparently when the liquid bridge touches the solid surface at $50 \tau$. The initial decrease in both $B_{x}$ and $B_{y}$ indicates an immediate shift of the contact line. The liquid-solid contact area shows a slight decrease compared to a slight increase in the case of $\beta=0.15$.

During the remaining stage of coalescence, as shown in Fig. 6(d), the moving contact line exhibits a larger receding velocity in the $x$-direction and a lower advancing velocity in the $y$-direction compared with those in the case of $\beta=0.15$. At the end of coalescence, the liquid-solid contact area in the case of $\beta=0.05$ is less than that in the case of $\beta=0.15$. When $B_{x}$ and $B_{y}$ are observed to be equal at $t_{\mathrm{a}}=117 \tau$, the nanodroplet transforms into a spherical-crown shape, marking the end of coalescence process. The velocity of the mass center $v_{\mathrm{c}}$ reaches the maximum value of $0.081 \sigma \tau^{-1}$, which is larger than that of $0.045 \sigma \tau^{-1}$ in the case of $\beta=0.15$.

\section{B. Restoration stage $\left(t_{\mathrm{a}}<t<t_{\mathrm{b}}\right)$}

\section{On surface with $\beta=0.15$}

With the height of the mass center $z_{\mathrm{c}}$ increasing continuously, the spherical-crown-shaped nanodroplet evolves towards a spherical shape. The contact line remains receding in both $x$ - and $y$-directions, leading to a decreasing liquid-solid contact area, i.e. dewetting process. It is important to note that the 
receding velocities of the moving contact line are lower in both $x$-and $y$-directions than those in the case of $\beta=0.05$ (see Figs. 6(c) and (d)). At $t_{\mathrm{b}}=220 \tau, v_{\mathrm{c}}$ decreases to zero, the contact line stops receding and the height of the mass center reaches its maximum value.

\section{On surface with $\beta=0.05$}

Similarly, as shown in Figs. 4(a) and (b), as the mass center of nanodroplet keeps rising, the upwards $v_{\mathrm{c}}$ gradually decreases with time because the rising nanodroplet is impeded by the adhesive force due to solid-fluid interaction. The contact line recedes in both $x$ - and $y$-directions and the liquid-solid contact area shrinks. As shown in Fig. 6, with both $B_{x}$ and $B_{y}$ decreasing, the nanodroplet restores towards a spherical shape. The magnitude of adhesive force, depending on the number of ls molecules, decreases with the reduction in liquid-solid contact area. At $t_{\mathrm{b}}=225 \tau$, both $B_{x}$ and $B_{y}$ decreases to zero and an observed upwards $v_{\mathrm{c}}$ of $0.055 \sigma \tau^{-1}$ is the jumping-off velocity.

\section{Oscillation stage / jumping stage $\left(t>t_{\mathrm{b}}\right)$}

\section{On surface with $\beta=0.15$}

The nanodroplet is pulled back by the adhesive force and re-spreads on the solid surface. The liquidsolid contact area then expands, as shown in Fig. 7(a), with the contact line advancing in both $x$ - and $y$ directions. Oscillation is seen lasting for a long time, as shown by the fluctuating $z_{\mathrm{c}}$ in the inset in Fig. 4(a). Consequently, the nanodroplet fails to jump up.

\section{On surface with $\beta=0.05$}

After detachment, the nanodroplet keeps rising and its $v_{c}$ decreases linearly (see Fig. 4(b)), suggesting a constant drag force by the vapor on the jumping nanodroplet. The vapor resistance as a surface force is different from the gravitational force as a bulk force. It is noteworthy that the former grows more and more significant than the latter with the droplet downsizing into nanoscale due to considerably large surfaceto-volume ratio. The present result supplements the earlier work that air friction has little influence on the jumping velocity of microdroplets. ${ }^{26}$

\section{Energy conversion analysis}


In the investigations of coalescence-induced nanodroplet jumping, the excess surface free energy

released $\left(\Delta E_{\mathrm{s}}\right)$ is defined as the changes in the surface free energy of liquid-solid and liquid-vapor interfaces.

$\Delta E_{\mathrm{s}}$ is expressed by Eq. (1) as:

$$
\Delta E_{\mathrm{s}}=\Delta A_{\mathrm{ls}} \sigma_{\mathrm{ls}}+\Delta A_{\mathrm{lv}} \sigma_{\mathrm{lv}}
$$

where $\Delta A_{\mathrm{ls}}$ and $\Delta A_{\mathrm{lv}}$ are the changes in areas of the liquid-solid and liquid-vapor interfaces, respectively; $\sigma_{\mathrm{ls}}$ and $\sigma_{\mathrm{lv}}$ are the liquid-solid and liquid-vapor interfacial tensions, respectively. ${ }^{47}$ For a planar liquidvapor interface, $\sigma_{\mathrm{lv}}$ is calculated from the integral of the difference between the normal and tangential components of pressure tensors ${ }^{48,49}$ where the pressure tensors can be obtained from the microscopic intermolecular potential force in molecular dynamics simulation. For example, the liquid-vapor surface tension at $T_{\mathrm{v}}=0.75 \varepsilon k_{\mathrm{B}}^{-1}$ is calculated to be $\sigma_{\mathrm{lv}}=1.10 \varepsilon / \sigma^{2} \cdot{ }^{47}$ For nanodroplets, however, $\Delta A_{\mathrm{ls}}$ and $\Delta A_{\mathrm{lv}}$ are difficult to be calculated in molecular dynamics simulation and $\sigma_{\mathrm{lv}}$ is dependent on the radius ${ }^{50}$ Therefore, a method is needed to evaluate the surface free energy of coalescing nanodroplet.

Figures. 7(c) and 7(d)show the evolutions of the changes in molecular potential energy of $1 \mathrm{~s}$ molecules $\left(\Delta E_{\mathrm{p}, \mathrm{ls}}\right)$, lv molecules $\left(\Delta E_{\mathrm{p}, \mathrm{lv}}\right)$ and 11 molecules $\left(\Delta E_{\mathrm{p}, \mathrm{ll}}\right)$. The change in the molecular potential energy of all liquid molecules ( $\Delta E_{\mathrm{p}}$ ) can be expressed by Eq. (2)

$$
\Delta E_{\mathrm{p}}=\Delta E_{\mathrm{p}, \mathrm{lv}}+\Delta E_{\mathrm{p}, \mathrm{ls}}+\Delta E_{\mathrm{p}, 11}
$$

The evolutions of $\Delta E_{\mathrm{p}}$ with time in both cases are plotted in Fig. 8. Furthermore, $\Delta E_{\mathrm{p}, \mathrm{lv}}=\Delta N_{\mathrm{lv}} e_{\mathrm{lv}}$, $\Delta E_{\mathrm{p}, \mathrm{ls}}=\Delta N_{1 \mathrm{~s}} e_{\mathrm{ls}}$ and $\Delta E_{\mathrm{p}, \mathrm{ll}}=\Delta N_{11} e_{\mathrm{ll}}$, where $e_{\mathrm{lv}}, e_{11}$ and $e_{\mathrm{ls}}$ are the average molecular potential energies per lv molecule, per 11 molecule and per ls molecule, respectively. With $n_{\mathrm{lv}}$ and $n_{\mathrm{ls}}$ representing the number densities per unit area of the liquid-vapor and liquid-solid interfaces, $\Delta N_{\mathrm{lv}}$ and $\Delta N_{\mathrm{ls}}$ can be expressed as $\Delta N_{\mathrm{lv}}=\Delta A_{\mathrm{lv}} n_{\mathrm{lv}}$ and $\Delta N_{\mathrm{ls}}=\Delta A_{\mathrm{ls}} n_{\mathrm{ls}}$, respectively. Equation (2) can be expressed as:

$$
\Delta E_{\mathrm{p}}=\Delta A_{\mathrm{ls}} n_{\mathrm{ls}} e_{\mathrm{ls}}+\Delta A_{\mathrm{lv}} n_{\mathrm{lv}} e_{\mathrm{lv}}-\Delta N_{11} e_{\mathrm{ll}}
$$


Moreover, since there exist no liquid-vapor phase-change, it is reasonably assumed that the number of all liquid molecules are not changed, i.e. $\Delta N_{\mathrm{ls}}+\Delta N_{\mathrm{lv}}+\Delta N_{\mathrm{ll}}=0$. Subsequently, the change of molecular potential energy $\left(\Delta E_{\mathrm{p}}\right)$ can be expressed as:

$$
\Delta E_{\mathrm{p}}=\Delta A_{\mathrm{lv}} n_{\mathrm{lv}}\left(e_{11}-e_{\mathrm{lv}}\right)+\Delta A_{\mathrm{ls}} n_{\mathrm{ls}}\left(e_{11}-e_{\mathrm{ls}}\right)
$$

The liquid-vapor interfacial tension can be understood at molecular level. Due to the existence of the liquid-vapor interface, the molecular potential energy of lv molecules is higher than that of 11 molecules. The transfer of liquid-vapor interfacial molecules into bulk liquid molecules results in the decrease in molecular potential energy i.e. the release of the excess surface free energy. Thus, the liquid-vapor interfacial tension can be obtained by calculating the release of the excess surface free energy per unit area of the liquid-vapor interface using Eq.(5)

$$
\sigma_{\mathrm{lv}}=n_{\mathrm{lv}}\left(e_{11}-e_{\mathrm{lv}}\right)
$$

For the system shown in Fig. 2, the number of lv molecules per unit area $\left(n_{\mathrm{lv}}\right)$ is calculated to be $1.52 \sigma^{-2}$. The average molecular potential energies per 11 molecule and per ls molecule are calculated to be $e_{11}=-5.69 \varepsilon$ and $e_{\mathrm{lv}}=-4.90 \varepsilon$, respectively. $n_{\mathrm{lv}}\left(e_{11}-e_{\mathrm{lv}}\right)$ is calculated to be $1.20 \varepsilon / \sigma^{2}$. This value is close to the liquid-vapor interfacial tension of $1.10 \varepsilon / \sigma^{2}$ at the same temperature obtained by the method based on pressure tensors. ${ }^{47}$ Similarly, the liquid-solid interface tension can be calculated by Eq. (6)

$$
\sigma_{\mathrm{ls}}=n_{\mathrm{ls}}\left(e_{\mathrm{ll}}-e_{\mathrm{ls}}\right)
$$

With Eqs. (5) and (6), we can see an equivalence between Eq. (1) and Eq. (4), presenting that the change in molecular potential energy is equal to the released surface free energy i.e. $\Delta E_{\mathrm{p}}=\Delta E_{\mathrm{s}}$.

Therefore, we can monitor the release of excess surface free energy by calculating the molecular potential energy of all liquid molecules. In both cases, $\Delta E_{\mathrm{p}}$ keeps deceasing in the coalescence stage and holds around $-1250 \varepsilon$ afterwards. We further examine the changes in the total kinetic energy of the nanodroplet $\left(\Delta E_{\mathrm{k}}\right)$ and its components in the $x$-, $y$ - and $z$-directions $\left(\Delta E_{\mathrm{k}, x}, \Delta E_{\mathrm{k}, y}\right.$ and $\left.\Delta E_{\mathrm{k}, z}\right)$, as shown in Fig. 9. Fig. 10 schematically summarizes the results. In the coalescence stage, for the non-jumping case, the mass center of the nanodroplet is much closer to the solid surface. A larger fraction of the nanodroplet could be pushed by the solid surface, leading to a larger bouncing force, as shown by the steeper variation of the 
momentum in the $z$-direction. ${ }^{21}$ Nevertheless, due to a downwards initial velocity and a shorter period of acceleration time, its maximum value of $v_{\mathrm{c}}$ is apparently lower than that in the jumping case.

In the coalescence stage, the maximum value of $\Delta E_{\mathrm{k}}$ in the jumping case is $220 \varepsilon$ while that in the non-jumping case is $196 \varepsilon$. Note that most of the kinetic energy is in the $x$-direction. In fact, only the kinetic energy in the $z$-direction, $\Delta E_{\mathrm{k}, \mathrm{z}}$, could contribute to the jumping-up. At the end of the coalescence stage, $\Delta E_{\mathrm{k}, \mathrm{z}}$ in the non-jumping case is only $\Delta E_{\mathrm{k}, \mathrm{z}}=25 \varepsilon$ while that in the jumping case is $\Delta E_{\mathrm{k}, \mathrm{z}}=44 \varepsilon$; the conversion efficiencies of the kinetic energy in the $z$-direction are $2.0 \%$ and $3.5 \%$, respectively. In the case of $\beta=0.05$, a slightly higher conversion efficiency and a slightly larger $\Delta E_{\mathrm{k}, \mathrm{z}}$ at the end of coalescence stage exceeds the viscous dissipation and interfacial dissipation. The lower conversion efficiency of $\Delta E_{\mathrm{k}, \mathrm{z}}$ is attributed to larger viscous dissipation due to larger shape change and the larger interfacial dissipation due to the stronger solid-fluid interaction and larger liquid-solid contact area.

In the restoration stage, the lower interfacial dissipation in the jumping case is demonstrated by the fast receding of the contact line and the quick decrease of the liquid-solid contact area, which leads to a value of the kinetic energy in the $z$-direction when the liquid-solid surface decreases to zero. Eventually the nanodroplet jumps up. In the jumping case $\Delta E_{\mathrm{k}, z}$ has a value of $41 \varepsilon$ and a conversion rate of $\Delta E_{\mathrm{k}, z} 3.2 \%$. This conversion rate is less than that of $6 \%$ for coalescence-induced microdroplet jumping predicted in earlier investigations ${ }^{4}$. Further simulations are conducted. We find that when $\beta=0.075$ the upwards velocity $z_{\mathrm{c}}$ and the liquid-solid contact area simultaneously decreases to zero, suggesting the critical condition between non-jumping and jumping.

\section{CONCLUSIONS}

Molecule dynamics simulations have been performed to investigate how solid surface free energy determines coalesced-nanodroplet jumping. We exhibit the dynamics of the moving contact line and energy conversion during the coalescence and jumping/non-jumping of nanodroplet. An interface detection method is proposed and the evolutions of the number of interfacial molecules and their molecular potential energies are analyzed. The excess surface free energy released and energy conversion efficiency are evaluated. 
We find that an increase in solid surface free energy ( $\beta$ from 0.05 to 0.15 ) essentially switches the coalesced-nanodroplet from jumping to non-jumping. With the solid surface free energy increasing, in the coalescence stage, better wetting status and stronger impingement result in a larger bouncing force. However, due to the downwards initial velocity and shorter acceleration time, the nanodroplet fails to acquire a higher upwards velocity. Moreover, larger liquid-solid contact area suggests a larger shape-change during the coalescence and an increase in viscous and interfacial dissipation. At the end of the coalescence stage, the nanodroplet obtained slightly lower kinetic energy in the jumping direction in the case of $\beta=0.15$ due to higher viscous dissipation. Finally, in the restoration stage, higher surface free energy leads to larger dissipation effect and lower kinetic energy in the jumping direction. Fundamentally, it is the coupled viscous and interfacial dissipation through the coalescence and restoration stages that eventually prevent the nanodroplet from jumping up. Our results address the importance of the liquid-solid surface interaction in the coalescence-induced jumping of nanodroplets in comparison with the jumping of microdroplets and the determination of the minimum size of jumping nanodroplets.

\section{ACKNOWLEDGEMENTS}

Financial supports from the National Natural Science Foundation of China (51406205), Joint PhD studentship of China Scholarship Council (CSC) and Queen Mary University of London and the Engineering and Physics Science Research Council (EPSRC) of the UK (EP/N001236/1, EP/N020472/1) are acknowledged.

\section{REFERENCES}

1 J. B. Boreyko and C. H. Chen, Phys. Rev. Lett. 103, 184501 (2009).

2 J. B. Boreyko and C. H. Chen, Phys. Fluids 22, 091110 (2010).

3 Y. Nam, H. Kim and S. Shin, Appl. Phys. Lett. 103, 161601 (2013).

4 R. Enright, N. Miljkovic, J. Sprittles, K. Nolan, R. Mitchell and E. N. Wang, ACS Nano 8, 10352 (2014).

5 F. Liu, G. Ghigliotti, J. J. Feng, C. H. Chen, J. Fluid Mech. 752, 39 (2014).

6 K. M. Wisdom, J. A. Watson, X. Qu, F. Liu, G. S. Watson and C. H. Chen, Proc. Natl. Acad. Sci. U. S. A.

110, 7992 (2013).

7 G. S. Watson, M. Gellender and J. A. Watson. Biofouling 30, 427 (2014).

8 Q. L. Zhang, M. He, J. Chen, J. J. Wang, Y. L. Song and L. Jiang, Chem. Commun. 49, 4516 (2013). 
9 J. B. Boreyko and P. C. Collier, ACS Nano 7, 1618 (2013).

10 N. Miljkovic, R. Enright, Y. Nam, K. Lopez, N. Dou, J. Sack and E. N. Wang, Nano Lett. 13, 179 (2013). 11 N. Miljkovic, R. Enright and E. N. Wang, ACS Nano 6, 1776 (2012).

12 C. J. Lv, P. F. Hao, Z. H. Yao, Y. Song, X. Zhang and F. He, Appl. Phys. Lett. 103, 021601 (2013).

13 C. J. Lv, P. F. Hao, Z. H. Yao and F. L. Niu, Langmuir 31, 2414 (2015).

14 K. Yanagisawa, M. Sakai, T. Isobe, S. Matsushita and A. Nakajima, Appl. Surf. Sci. 315, 212 (2014).

15 J. Liu, H. Y. Guo, B. Zhang, S. S. Qiao, M. Z. Shao, X. R. Zhang, X. Q. Feng, Q. Y. Li, Y. L. Song, L. Jiang and J. J. Wang, Angew. Chem. Int. Ed. 55, 4265 (2016).

16 S. N. Zhang, J. Y. Huang, Y. X. Tang, S. H. Li, M. Z. Ge, Z. Chen, K. Q. Zhang and Y. K. Lai, Small 00687 (2016).

17 X. M. Chen, R. S. Patel, J. A. Weibel and S. V. Garimella, Sci. Rep. 6, 18649 (2016).

18 A. Aili, H. Li, M. H. Alhosani and T. Zhang, ACS Appl. Mater. Interfaces 8, 21776 (2016).

19 F. Chu, X. Wu, B. Zhu and X. Zhang, Appl. Phys. Lett. 108, 194103 (2016).

20 K. Wang, Q. Liang, R. Jiang, Y. Zheng, Z. Lan and X. H. Ma, RSC Advances 6, 99314 (2016).

21 X. Qu, J. B. Boreyko, F. Liu, R. L. Agapov, N. V. Lavrik, S. T. Retterer, J. J. Feng, C. P. Collier and C. H. Chen, Appl. Phys. Lett. 106, 221601 (2015).

22 J. Tian, J. Zhu, H. Y. Guo, J. Li, X. Q. Feng and X. F. Gao, J. Phys. Chem. Lett. 5, 2084 (2014).

23 K. Zhang, F. Liu, A. J. Williams, X. Qu, J. J. Feng and C. H. Chen, Phys. Rev. Lett. 115, 074502 (2015).

24 F. C. Wang, F. Yang and Y. P. Zhao, Appl. Phys. Lett. 98, 053112 (2011).

25 T. Q. Liu, W. Sun, X. Y. Sun and H. R. Ai, Colloids Surf. A 414, 366 (2012).

26 B. L. Peng, S. F. Wang, Z. Lan, W. Xu, R. F. Wen and X. H. Ma, Appl. Phys. Lett. 102, 151601 (2013).

27 X. L. Liu, P. Cheng and X. J. Quan, Int. J. Heat Mass Transfer 73, 195 (2014).

28 X. L. Liu and P. Cheng, Int. Commun. Heat Mass Transfer 64, 7 (2015).

29 S. Farokhirad, J. F. Morris and T. Lee, Phys. Fluids 27, 102102 (2015).

30 Z. Liang and P. Keblinski, Appl. Phys. Lett. 107, 143105 (2015).

31 Y. Nam, D. Seo, C. Lee and S. Shin, Soft Matter 11, 154 (2015).

32 Y. Shi, G. H. Tang and H. H. Xia, Int. J. Heat Mass Transfer 88, 445 (2015).

33 Y. P. Cheng, J. L. Xu and Y. Sui, Int. J. Heat Mass Transfer 95, 506 (2016).

34 M. He, X. Zhou, X. Zeng, D. Cui, Q. Zhang, J. Chen and L. Jiang, Soft Matter 8, 6680 (2012).

35 H. Cha, C. Xu, J. Sotelo, J. M. Chun, Y. Yokoyama, R. Enright and N. Miljkovic, Phys. Rev. Fluids 1, 064102 (2016).

36 R. D. Narhe and D. A. Beysens, Langmuir 23, 6486 (2007).

37 C. Andrieu and A. Doucet, J R. Stat. Soc. Series B Stat. Methodol. 64, 827 (2002).

38 S. Herminghaus, M. Brinkmann and R. Seemann, Annu. Rev. Mater. Res. 38, 101 (2008).

39 S. Maruyama, Advances in Numerical Heat Transfer (Taylor \& Francis Press, 2000)

40 P. Yi, D. Poulikakos, J. Walther and G. Yadigaroglu, Int. J. Heat Mass Transfer 45, 2087 (2002).

41 P. A. Thompson and M. O. Robbins, Phys. Rev. A 41, 6830 (1990).

42 P. A. Thompson and S. M. Troian, Nature 389, 360 (1997). 
43 Q. Sheng, J. Sun, Q. Wang, W. Wang and H. S. Wang, Sci. Rep. 6, 30764 (2016). 44 J. Sun and H. S. Wang, Sci. Rep. 6, 35003 (2016).

45 P. R. Wolde and D. Frenkel, J. Chem. Phys. 109, 9901 (1998).

46 S. Werth, S. V. Lishchuk, M. Horsch and H. Hasse, Phy. A 392, 2359 (2013).

47 J. G. Weng, S. Park, J. R. Lukes and C. L. Tien, J. Chem. Phys. 113, 5917 (2000).

48 S. H. Park, J. G. Weng and C. L. Tien, Int. J. Heat Mass Transfer 44, 1849 (2001).

49 M. J. P. Nijmeijer, A. F. Bakker, C. Bruin and J. H. Sikkenk, J. Chem. Phys. 89, 3789 (1988).

50 H.M. Lu and Q. Jiang, Langmuir 21, 779 (2005). 


\section{FIGURES}

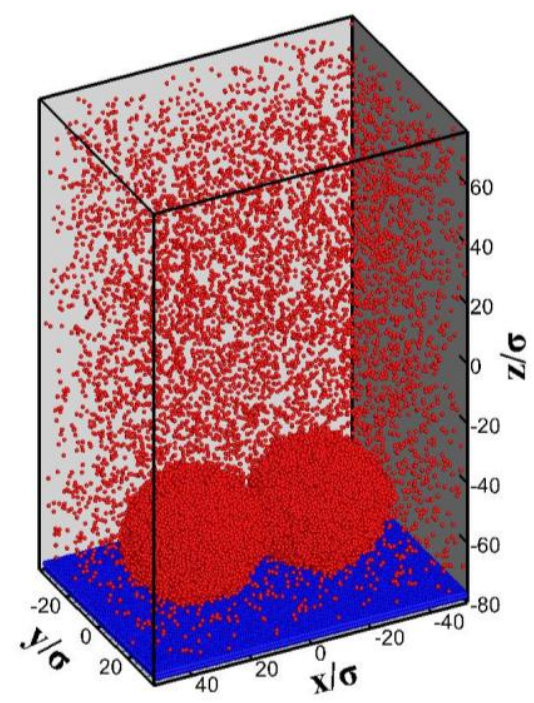

FIG. 1. Schematic of the simulation system. Fluid molecules are in red and solid atoms are in blue. The size of the simulation box is $l_{x} \times l_{y} \times l_{z}=105 \sigma \times 74 \sigma \times 155 \sigma$ and the radius of the nanodroplet is ca. $25 \sigma$. 

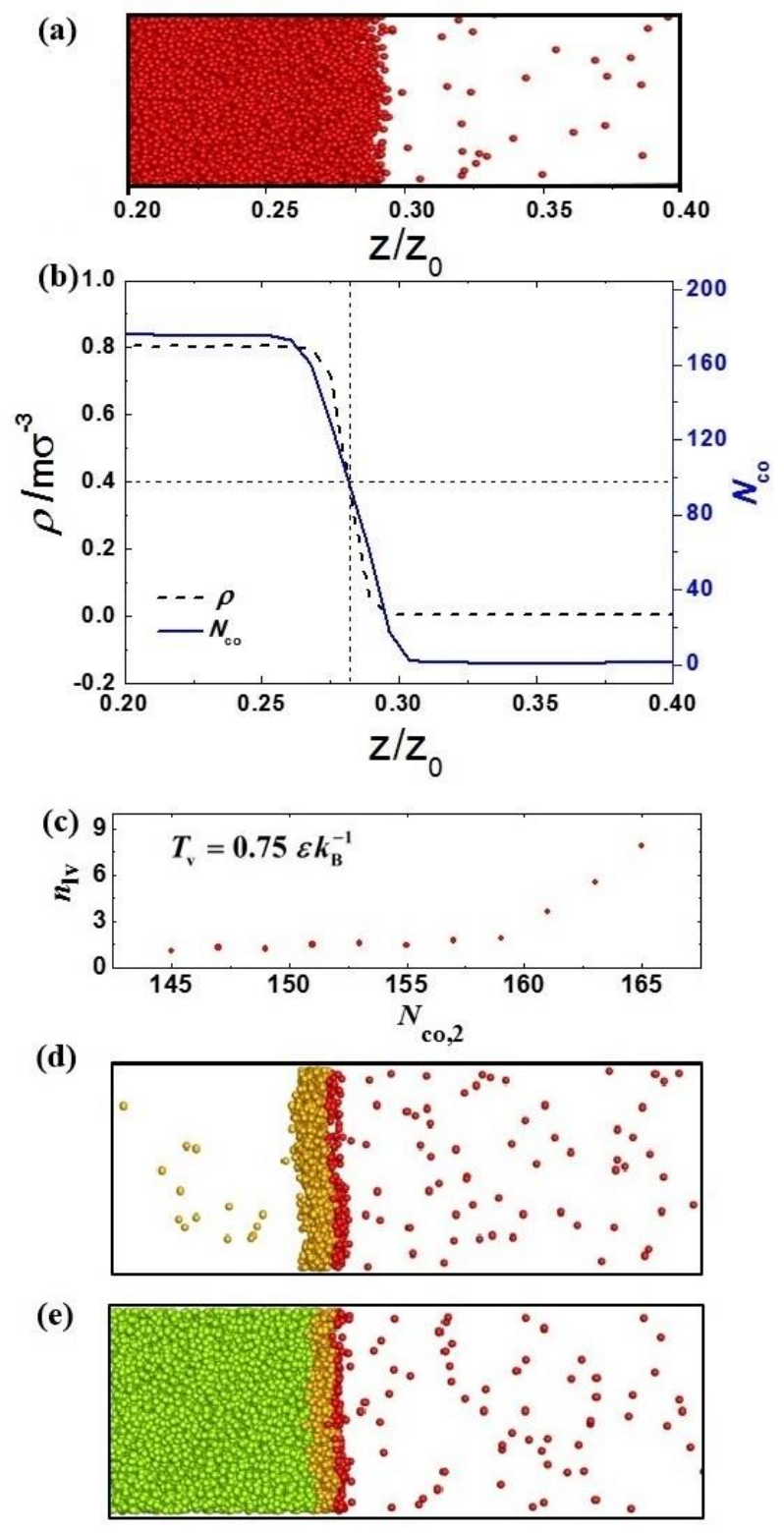

FIG. 2. Determination of the liquid-vapor interface. (a) Part of the liquid-vapor interface of a liquid-vapor equilibrium system; (b) Distributions of the density $\rho$ and coordination number $N_{\text {co }}$ along the normal to the liquid-vapor interface; (c) Dependence of the number density per unit area of detected liquid-vapor molecules $n_{\mathrm{lv}}$ on the critical coordination number $\left(N_{\mathrm{co}, 2}\right)$; Snapshots of detection results under different values of (d) $N_{\mathrm{co}, 2}=162$ and (e) $N_{\mathrm{co}, 2}=158$. The vapor molecules, liquid-vapor interfacial molecules and bulk liquid molecules are shown in red, yellow and green, respectively. 

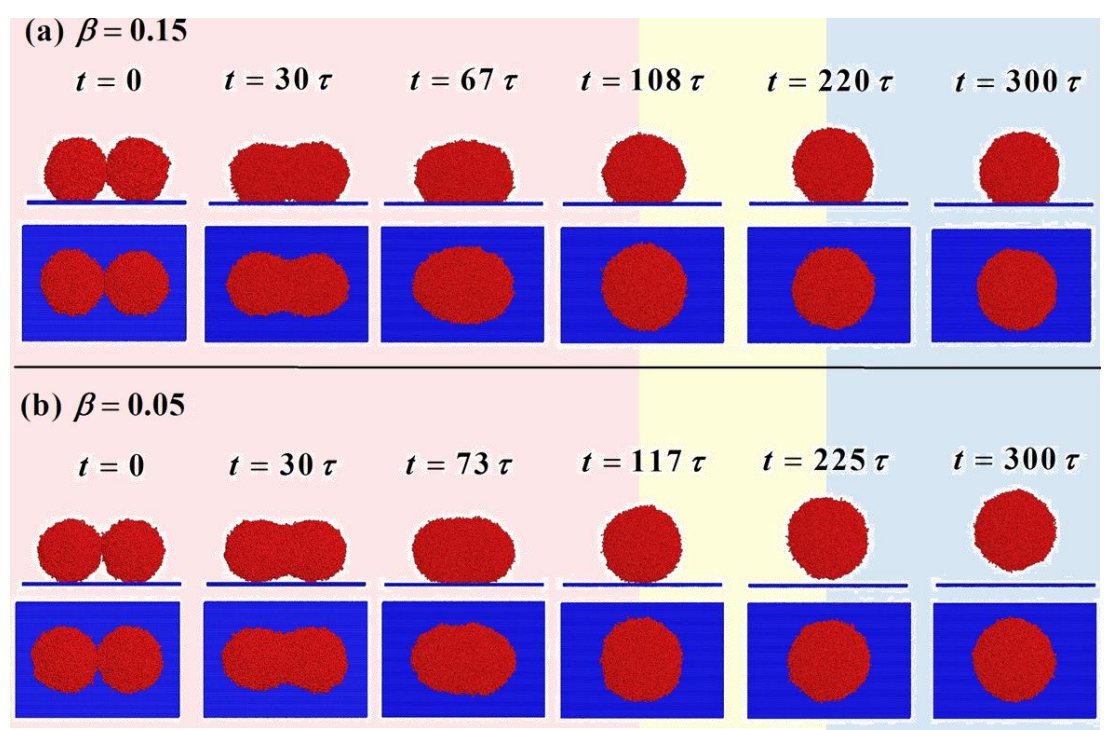

FIG. 3. Snapshots (side and top views) of coalescences of nanodroplet on the surfaces with (a) $\beta=0.15$ and (b) $\beta=0.05$ at different times. The coalescence, restoration and oscillation/jumping stages are shown in light red, yellow and blue background, respectively. The time at the end of coalescence stage is $t_{\mathrm{a}}$. The time at the end of restoration stage is $t_{\mathrm{b}}$. 

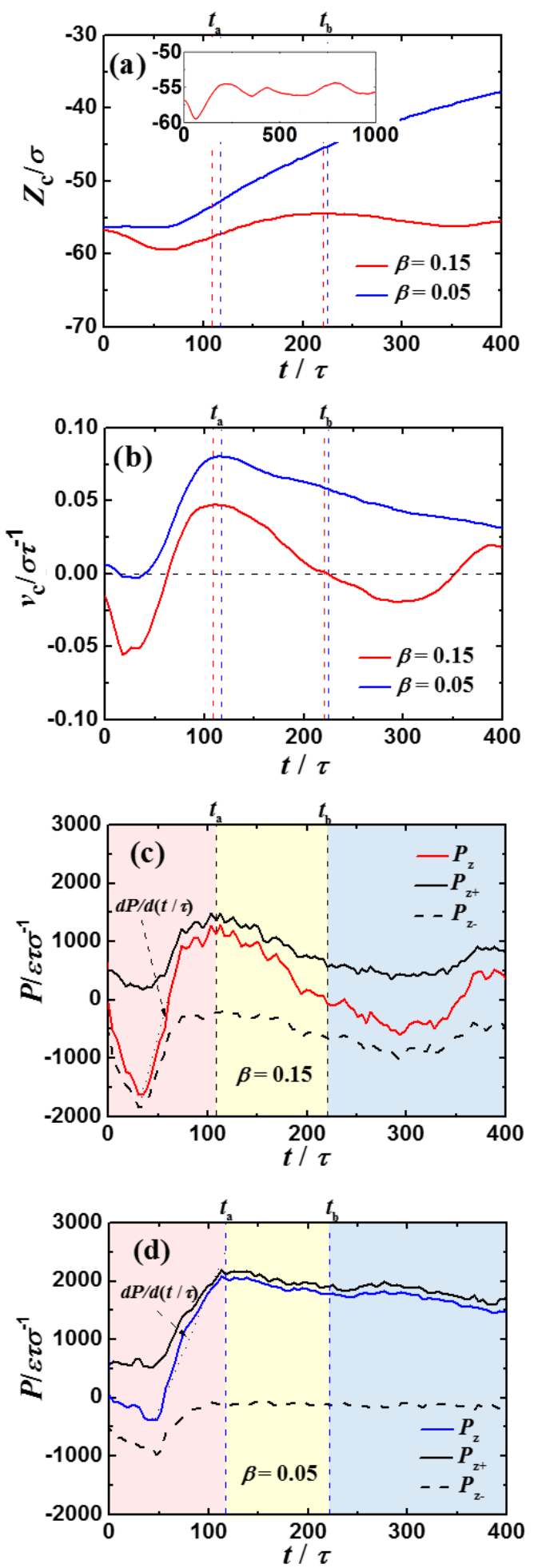

FIG. 4. Time-evolutions of (a) the position of the mass center $z_{\mathrm{c}}$ and (b) the velocity of the mass center $v_{\mathrm{c}}$ for the nanodroplet on the surfaces with $\beta=0.15$ and $\beta=0.05$. The characteristic times are indicated in red ( $\beta=0.15$ ) and blue ( $\beta=0.05$ ) dash vertical lines. Time-evolutions of the total momentum in the $z$-direction $P_{z}$, and its components in $+z$-direction $P_{z+}$ and $z$-direction $P_{z-}$ on the surfaces with (c) $\beta=0.15$ and (d) $\beta=0.05$. 


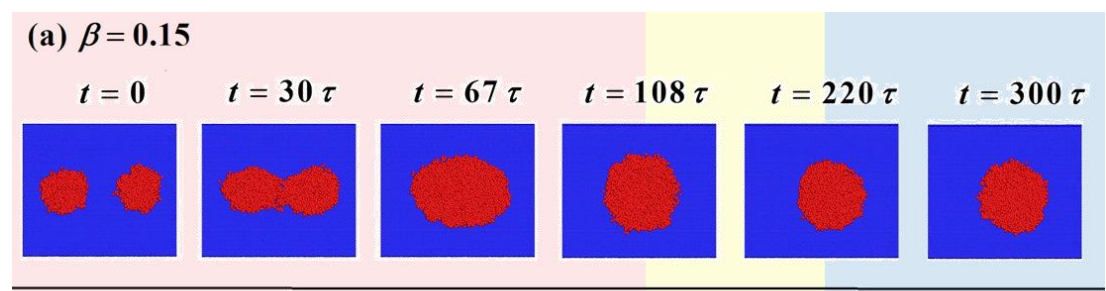

(b) $\beta=0.05$

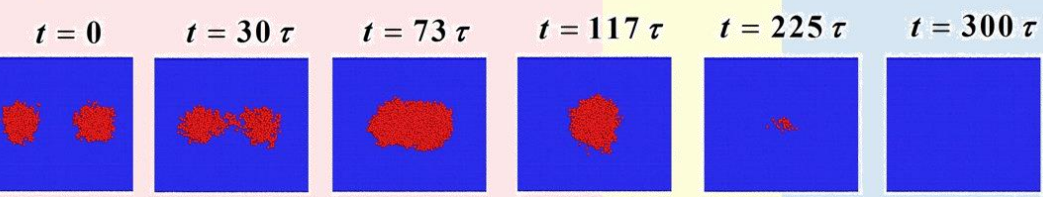

FIG. 5. Snapshots (top view) of the liquid-solid interfacial molecules with (a) $\beta=0.15$ and (b) $\beta=0.05$ at different times. The coalescence, restoration and oscillation/jumping stages are shown in light red, yellow and blue background, respectively. The time at the end of coalescence stage is $t_{\mathrm{a}}$. The time at the end of restoration stage is $t_{\mathrm{b}}$. 

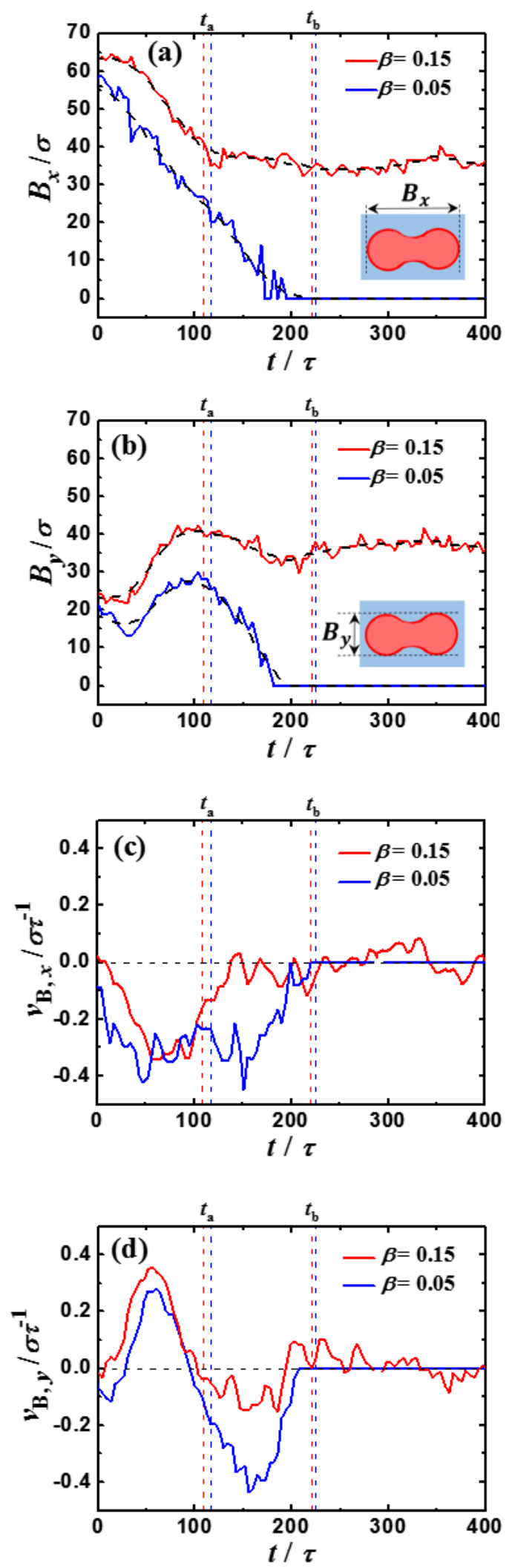

FIG. 6. Time-evolutions of size scales of the liquid-solid contact area (a) in the $x$-direction $B_{x}$ and (b) in the $y$-direction $B_{y}$ on the solid surfaces with $\beta=0.15$ and $\beta=0.05 . B_{x}$ and $B_{y}$ are measured between the extremes of the liquid-solid interface as shown in the inset. Time-evolutions of the moving velocities of the liquid-solid contact area (c) in the $x$-direction $v_{\mathrm{B}, x}$ and (d) in the $y$-direction $v_{\mathrm{B}, y}$ on the solid surfaces with $\beta=0.15$ and $\beta=0.05$. The characteristic times are indicated by red $(\beta=0.15)$ and blue $(\beta=0.05)$ dash vertical lines. 

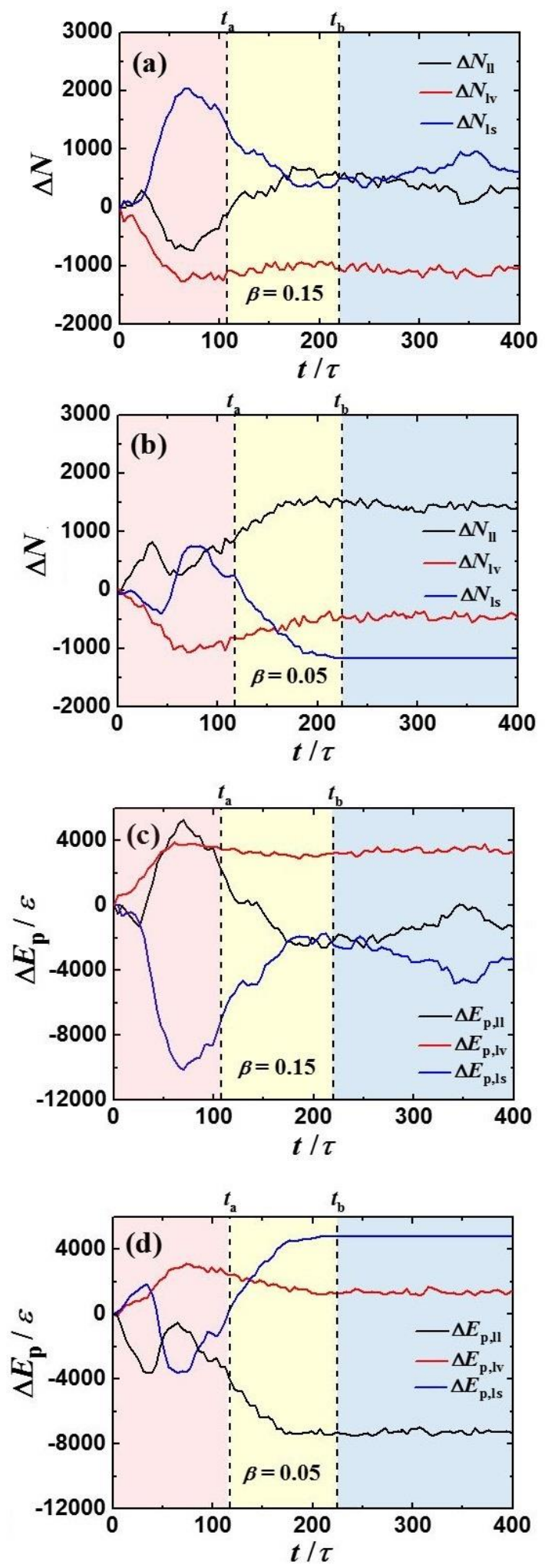

FIG. 7. Time-evolutions of number differences of bulk liquid molecules $\Delta N_{11}$, liquid-vapor interfacial molecules $\Delta N_{\mathrm{lv}}$ and liquid-solid interfacial molecules $\Delta N_{\mathrm{sl}}$ on the solid surfaces with (a) $\beta=0.15$ and (b) $\beta=0.05$. Time-evolutions of potential energy differences of bulk liquid molecules $\Delta E_{\mathrm{p}, \mathrm{ll}}$, liquid-vapor interfacial molecules $\Delta E_{\mathrm{p}, \mathrm{lv}}$ and liquid-solid interfacial molecules $\Delta E_{\mathrm{p}, \mathrm{sl}}$ on the surfaces with (c) $\beta=0.15$ and (d) $\beta=0.05$. The coalescence, restoration and oscillation/jumping stages are shown in light red, yellow and blue background, respectively. The characteristic times are indicated by dash vertical lines. 


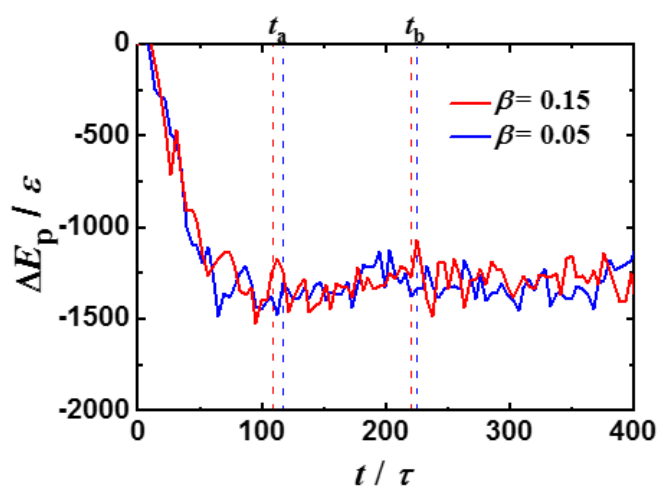

FIG. 8. Time-evolutions of the total potential energy difference $\Delta E_{\mathrm{p}}$ of the nanodroplet on the solid surfaces with $\beta=0.15$ and $\beta=0.05$. The characteristic times are indicated by red $(\beta=0.15)$ and blue ( $\beta=0.05$ )dash vertical lines. 

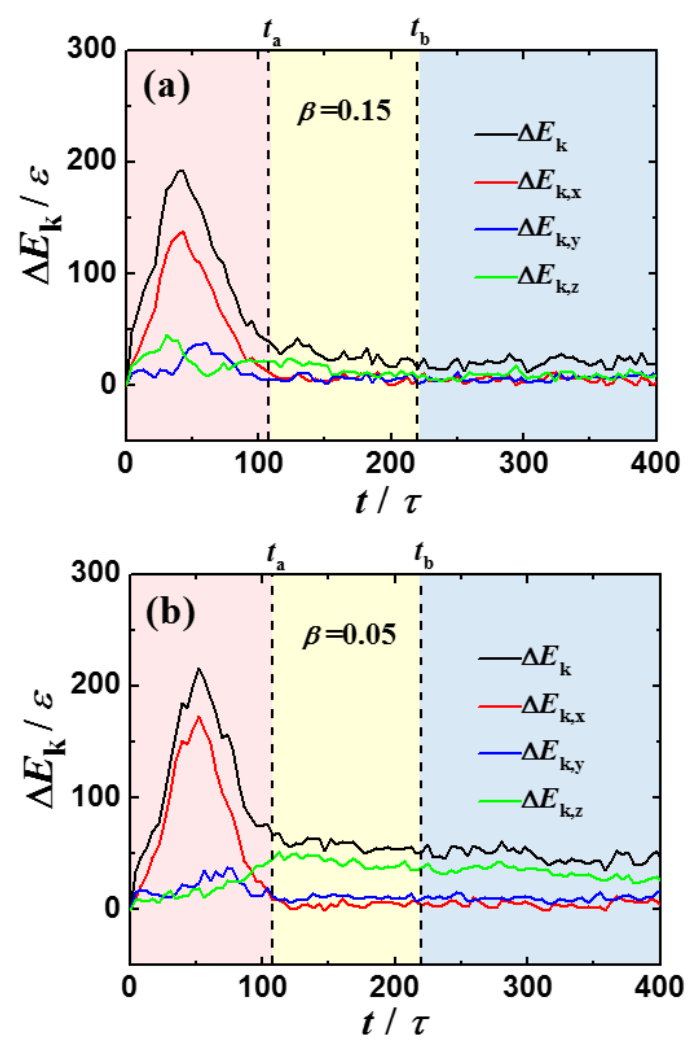

FIG. 9. Time-evolutions of kinetic energy differences of the nanodroplet in the $x$-direction $\Delta E_{\mathrm{k}, x}, y$-direction $\Delta E_{\mathrm{k}, y}$ and $z$-direction $\Delta E_{\mathrm{k}, z}$ on the solid surfaces with (a) $\beta=0.15$ and (b) $\beta=0.05$. The coalescence, restoration and oscillation/jumping stages are shown in light red, yellow and blue background, respectively. The characteristic times are indicated by dash vertical lines. 


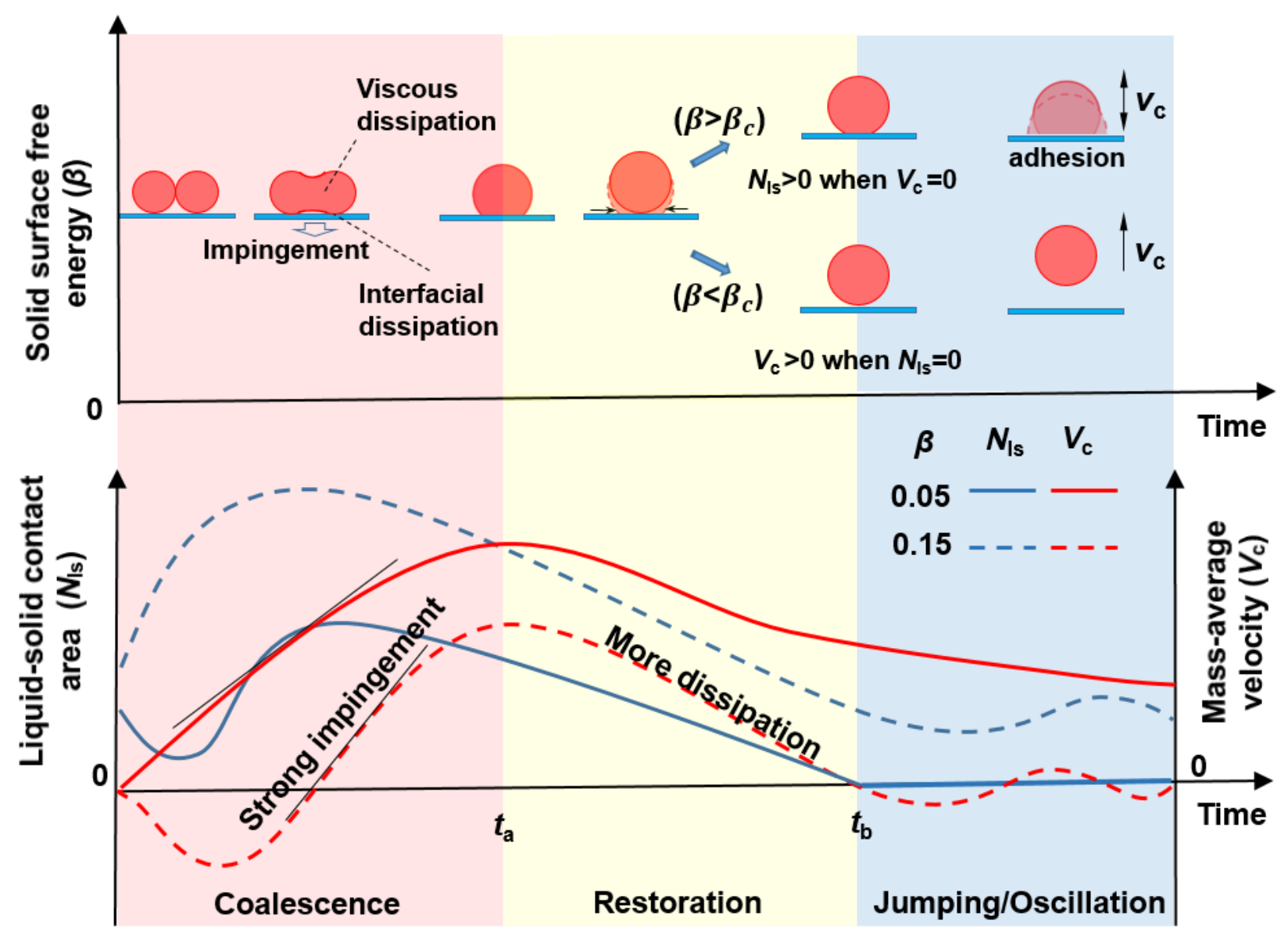

FIG. 10. Schematic presentation of the mechanism of solid surface free energy on coalescence-induced nanodroplet jumping. The coalescence, restoration and oscillation/jumping stages are shown in light red, yellow and blue background, respectively. $\beta_{c}$ is the critical value of solid surface free energy. 